\title{
NONUNIFORM MOTION OF AN EDGE DISLOCATION IN AN ANISOTROPIC SOLID. I*
}

\author{
By \\ XANTHIPPI MARKENSCOFF AND LUQUN NI ${ }^{\dagger}$ \\ University of California Santa Barbara
}

\begin{abstract}
The two-dimensional problem of the nonuniform motion of an edge dislocation in an anisotropic solid (regular hyperbolic case) is solved by means of Laplace transforms with inversion according to the Cagniard-de Hoop technique. The solution is also evaluated asymptotically at the saddle points on the Cagniard-de Hoop contour which lies on a multi-sheet Riemann surface, the singular points of which are examined in detail also in connection to the slowness surface. The stress field is square root singular near the wavefront for a motion of constant velocity starting from rest, and $2 / 3$ singular near the cusp-tips. For general nonuniform motion the stress at the wavefront is obtained as well and an example is given for a motion starting with constant acceleration.
\end{abstract}

Introduction. Uniform motion of dislocations in anisotropic solids has been studied in the past by Sáenz [1], Bullough and Bilby [2], Teutonico [3] and others. Nonuniform motion of dislocations, however, does not appear to the authors to have been yet considered by other investigators. For isotropic solids, Eshelby [4] was the first to give a solution for a screw dislocation moving nonuniformly. For more complete references on moving dislocations the reader is referred to the review article by Weertman and Weertman [5]. More recent references include Markenscoff and Clifton [6, 7] for nonuniformly moving edge dislocation and dislocation loops.

In the present work we consider the two-dimensional problem for the nonuniform motion of an edge dislocation with axis parallel to a cubic axis and moving along a cubic axis (of a cubic crystal) or an edge dislocation with axis normal to a symmetry plane of a transversely isotropic solid (hexagonal symmetry) as, for instance, an edge dislocation gliding on the basal plane of a hexagonal crystal in a direction of closest packing. The common case of an edge dislocation in a rock-salt type structure moving along a face-diagonal axis also falls within this category. We only consider here the regular

\footnotetext{
* Received January 24, 1983. Present address of the second author: Institute of Mathematics, Academia Sinica, Beijing (Peking), China.

${ }^{\dagger}$ The authors are grateful to Professor R. Burridge for valuable suggestions. This research was supported by the Solid Mechanics Program of the National Science Foundation through a grant to the University of California, Santa Barbara.
} 
hyperbolic case of the slowness cone [8]. The singular one is considered in part II of this research. Our methodology is also expandable to any two-dimensional problem which would occur when the dislocation is normal to a symmetry plane of the material. For a detailed discussion on this issue we refer to Teutonico [9].

The solution to the dynamic problem is obtained by the use of Laplace transforms in time and space, and the inversion by means of the Cagniard-de Hoop technique. In contrast to the isotropic case, the Cagniard-de Hoop contour lies now on a multi-sheet Riemann surface, the singular points of which are examined in detail. In order to obtain the wavefront behavior explicitly the solution is evaluated asymptotically near the saddle-points of the Cagniard-de Hoop contour which can also be found from a geometric construction. The wave-surface includes cusps which are examined in detail in relation to the corresponding points on the slowness-surface and the Cagniard-de Hoop contour. The asymptotic analysis is obtained for constant velocity motion (starting from rest) and for general nonuniform motion with a specific example worked-out for a motion starting with constant acceleration. In the constant velocity case the stress is square-root singular near the wavefront and $3 / 2$ singular near the cusp-tips.

I. General nonuniform motion of an edge dislocation in a cubic crystal. Let us consider a straight infinitely-long edge dislocation aligned parallel to the $x_{3}$ coordinate axis which coincides with the edge of a cubic crystal. The dislocation is at rest and at time $t=0$ it starts moving nonuniformly on the slip plane $x_{2}=0$ according to $x=l(t)$ or equivalently $t=\zeta(\xi)$, where $l(t)$ is any monotonically increasing function. Similarly as with the problem of the motion of an edge dislocation in an isotropic medium [6], the problem is reduced to one on the half-space $x_{2}>0$ and is equivalent to the superposition of the following two problems:

Problem I. The static problem with the dislocation being at the origin $x_{1}=0$.

Problem II. We seek the displacements $u_{1}\left(x_{1}, x_{2}, t\right)$ and $u_{2}\left(x_{1}, x_{2}, t\right)$ which satisfy the differential equations

$$
\begin{aligned}
& C_{11} \frac{\partial^{2} u_{1}}{\partial x_{1}^{2}}+C_{66} \frac{\partial^{2} u_{1}}{\partial x_{2}^{2}}+\left(C_{66}+C_{12}\right) \frac{\partial^{2} u_{2}}{\partial x_{1} \partial x_{2}}=\rho \frac{\partial^{2} u_{1}}{\partial t^{2}}, \\
& C_{66} \frac{\partial^{2} u_{2}}{\partial x_{1}^{2}}+C_{22} \frac{\partial^{2} u_{2}}{\partial x_{2}^{2}}+\left(C_{66}+C_{12}\right) \frac{\partial^{2} u_{1}}{\partial x_{1} \partial x_{2}}=\rho \frac{\partial^{2} u_{2}}{\partial t^{2}}
\end{aligned}
$$

with boundary conditions at $x_{2}=0$ :

$$
\begin{aligned}
u_{1}\left(x_{1}, 0, t\right) & =\frac{\Delta u}{2}\left[H\left(x_{1}-l(t)\right)-H\left(x_{1}\right)\right] \\
\sigma_{22}\left(x_{1}, 0, t\right) & \equiv C_{22} \frac{\partial u_{2}}{\partial x_{2}}+C_{12} \frac{\partial u_{1}}{\partial x_{1}}=0
\end{aligned} \quad \text { for all } t \geqslant 0,
$$

the quantity $\Delta u$ denoting the relative displacement (Burgers vector).

We should note that for a cubic material the elastic coefficient $C_{22}$ is equal to $C_{11}$, but it is different for transversely isotropic solids. 
The solution to the static Problem I may be found in Eshelby [10, 11] or in Willis [12], while for the solution to Problem II we use Laplace transforms in time and space, as presented in the sequel.

II. Solution by Laplace transforms. In order to solve the partial differential equations (1) with the boundary conditions (2) we use a Laplace transform in time:

$$
\hat{u}_{i}\left(x_{1}, x_{2}, s\right)=\int_{0}^{\infty} u_{i}\left(x_{1}, x_{2}, t\right) e^{-s t} d t
$$

and a two-sided Laplace transform in $x_{1}$

$$
U_{i}\left(\lambda, x_{2}, s\right)=\int_{-\infty}^{\infty} \hat{u}_{i}\left(x_{1}, x_{2}, s\right) e^{-s \lambda x_{1}} d x_{1}
$$

so that (1) and (2) yield the system of differential equations:

$$
\begin{aligned}
& C_{11} s^{2} \lambda^{2} U_{1}+C_{66} \frac{\partial^{2} U_{1}}{\partial x_{2}^{2}}+\left(C_{66}+C_{12}\right) s \lambda \frac{\partial U_{2}}{\partial x_{2}}=\rho s^{2} U_{1}, \\
& C_{66} s^{2} \lambda^{2} U_{2}+C_{22} \frac{\partial^{2} U_{2}}{\partial x_{2}^{2}}+\left(C_{66}+C_{12}\right) s \lambda \frac{\partial U_{1}}{\partial x_{2}}=\rho s^{2} U_{2}
\end{aligned}
$$

with boundary conditions at $x_{2}=0$ :

$$
\begin{gathered}
U_{1}(\lambda, 0, s)=\frac{-\Delta u}{2 s} \int_{0}^{\infty} e^{-s(\zeta(\xi)+\lambda \xi)} d \xi \\
s \lambda C_{21} U_{1}(\lambda, 0, s)+\left.C_{22} \frac{\partial U_{2}}{\partial x_{2}}\left(\lambda, x_{2}, s\right)\right|_{x_{2}=0}=0 .
\end{gathered}
$$

To solve the system of equations (3) we set

$$
\vec{W}=\left[U_{1}, \frac{\partial U_{1}}{\partial x_{2}}, \frac{\partial U_{2}}{\partial x_{2}}, U_{2}\right]^{T}
$$

so that (3) reduce to

$$
\frac{d \vec{W}}{d x_{2}}=\left|\begin{array}{cccc}
0 & 1 & 0 & 0 \\
-\frac{\left(C_{11} \lambda^{2}-\rho\right) s^{2}}{C_{66}} & 0 & -\frac{C_{66}+C_{12}}{C_{66}} s \lambda & 0 \\
0 & -\frac{C_{66}+C_{12}}{C_{22}} s \lambda & 0 & \frac{C_{66} \lambda^{2}-\rho}{C_{22}} s^{2} \\
0 & 0 & 1 & 0
\end{array}\right| \vec{W} .
$$

Solving (5) we find

$$
\begin{aligned}
& U_{1}\left(\lambda, x_{2}, s\right)=\sum_{i=1}^{4} A_{i}(\lambda, s) e^{\mu_{i} x_{2}}, \\
& U_{2}\left(\lambda, x_{2}, s\right)=\sum_{i=1}^{4} B_{i}(\lambda, s) e^{\mu_{i} x_{2}}
\end{aligned}
$$


where the constants $A_{i}$ and $B_{i}$ are determined from the boundary conditions (4) and the radiation conditions at infinity

$$
U_{1}, U_{2}, \frac{\partial U_{1}}{\partial x_{2}}, \frac{\partial U_{2}}{\partial x_{2}} \rightarrow 0, \quad \text { as } x_{2} \rightarrow \infty .
$$

The exponents $\mu_{i}$ are the roots (eigenvalues) of the determinantal equation

$$
\begin{aligned}
\Delta= & |A-\mu I| \\
= & \mu^{4}-\left[\left(\frac{\rho-C_{11} \lambda^{2}}{C_{66}}+\frac{\rho-C_{66} \lambda^{2}}{C_{22}}\right) s^{2}+\frac{\left(C_{66}+C_{12}\right)^{2}}{C_{22} C_{66}} s^{2} \lambda^{2}\right] \mu^{2} \\
& +\frac{\left(\rho-C_{11} \lambda^{2}\right)\left(\rho-C_{66} \lambda^{2}\right)}{C_{22} C_{66}} s^{4} \\
= & 0
\end{aligned}
$$

which may be written as

$$
\begin{aligned}
\Delta= & \frac{s^{4}}{C_{22} C_{66}}\left\{C_{22} C_{66}\left(\frac{\mu}{s}\right)^{4}+\left(\frac{\mu}{s}\right)^{2}\left[C_{22}\left(C_{11} \lambda^{2}-\rho\right)+C_{66}\left(C_{66} \lambda^{2}-\rho\right)\right.\right. \\
- & \left.\quad-\left\{\left(C_{66}+C_{12}\right)^{2} \lambda^{2}\right]+\left(\rho-C_{11} \lambda^{2}\right)\left(\rho-C_{66} \lambda^{2}\right)\right\} \\
\equiv & \frac{s^{4}}{C_{22} C_{66}} \Omega\left(\lambda, \frac{\mu}{s}\right)=0
\end{aligned}
$$

where $\Omega(\lambda, \eta) \equiv \Omega(\lambda, \mu / s)$ is the slowness surface for the crystal symmetry class under consideration [8].

Equation (9) has the four roots

$$
\eta(\lambda)= \pm \frac{1}{\sqrt{2}} \sqrt{p \pm q}
$$

where $\sqrt{p \pm q}$ denotes the branch of $[p \pm q]^{1 / 2}$ with non-negative real part,

$$
\begin{aligned}
& p(\lambda)=\frac{\rho-C_{11} \lambda^{2}}{C_{66}}+\frac{\rho+\lambda^{2} C_{12}\left(2+C_{12} / C_{66}\right)}{C_{22}}, \\
& q(\lambda)=\left[p^{2}(\lambda)-\frac{4\left(\rho-C_{11} \lambda^{2}\right)\left(\rho-C_{66} \lambda^{2}\right)}{C_{22} C_{66}}\right]^{1 / 2} \quad \text { (with non-negative real part). }
\end{aligned}
$$

From (6), (4) and (7) the solution for $U_{1}\left(\lambda, x_{2}, s\right), U_{2}\left(\lambda, x_{2}, s\right)$ is obtained:

$$
\begin{aligned}
& U_{1}\left(\lambda, x_{2}, s\right)=U_{1}(\lambda, 0, s)\left[D_{1}^{+}(\lambda) \exp \left(-s x_{2} \sqrt{\frac{p+q}{2}}\right)+D_{1}^{-}(\lambda) \exp \left(-s x_{2} \sqrt{\frac{p-q}{2}}\right)\right], \\
& U_{2}\left(\lambda, x_{2}, s\right)=U_{1}(\lambda, 0, s)\left[D_{2}^{+}(\lambda) \exp \left(-s x_{2} \sqrt{\frac{p+q}{2}}\right)+D_{2}^{-}(\lambda) \exp \left(-s x_{2} \sqrt{\frac{p-q}{2}}\right)\right],
\end{aligned}
$$


with

$$
\begin{gathered}
D_{1}^{ \pm}(\lambda)=\frac{1}{2} \pm \frac{\left(C_{22}-C_{66}\right) \rho-\lambda^{2}\left(C_{11} C_{22}-C_{12}^{2}\right)}{2 C_{22} C_{66} q} \\
D_{2}^{ \pm}(\lambda)=\frac{ \pm \lambda}{q \sqrt{2}(p \pm q)}\left\{\frac{\rho}{C_{22}^{2} C_{66}}\left(C_{66} C_{21}+2 C_{22} C_{66}+C_{22} C_{12}\right)\right. \\
-\frac{\lambda^{2}}{C_{22}^{2} C_{66}}\left[C_{21}\left(C_{66}^{2}-C_{11} C_{22}\right)+2 C_{11} C_{22}\left(C_{66}+C_{12}\right)\right. \\
\left.\left.\quad-C_{12}\left(C_{66}+C_{12}\right)^{2}\right] \pm \frac{C_{21}}{C_{22}} q\right\}
\end{gathered}
$$

and $U_{1}(\lambda, 0, s)$ given by (4-1).

We may note here that in the isotropic case for which $C_{66}=\frac{1}{2}\left(C_{11}-C_{12}\right)$ and $C_{11}=C_{22}, q=\rho\left(C_{22}-C_{66}\right) / C_{22} C_{66}$ and the expressions (10) yields $\pm \sqrt{\rho / C_{11}-\lambda^{2}}$, and $\pm \sqrt{\rho / C_{66}-\lambda^{2}}$, as expected [6].

We discuss now the Riemann surface for the multi-valued function $\eta(\lambda)=$ $\pm \sqrt{p \pm q} / \sqrt{2}$ which comprises four sheets. The Riemann surface for anisotropic materials has been analyzed by Burridge [13]. Each branch of $\eta(\lambda)$ is real on a segment of the $\operatorname{Re} \lambda$ axis containing the origin and on the segment $\left(-\sqrt{\rho / C_{11}}, \sqrt{\rho / C_{11}}\right)$ [13]. On the segment $\left(-\sqrt{\rho / C_{11}}, \sqrt{\rho / C_{11}}\right)$ let

$$
\eta_{1}=\frac{+1}{\sqrt{2}} \sqrt{p-q}, \quad \eta_{2}=\frac{+1}{\sqrt{2}} \sqrt{p+q}, \quad \eta_{3}=\frac{-1}{\sqrt{2}} \sqrt{p-q}, \quad \eta_{4}=\frac{-1}{\sqrt{2}} \sqrt{p+q}
$$

Then, according to Burridge, the branches $\eta^{\mathrm{I}}(\lambda), \eta^{\mathrm{II}}(\lambda), \eta^{\mathrm{III}}(\lambda)$, and $\eta^{\mathrm{IV}}(\lambda)$ are defined by analytic continuation from the segment $\left(-\sqrt{\rho / C_{11}}, \sqrt{\rho / C_{11}}\right)$ of the $\operatorname{Re} \lambda$ axis, without $\lambda$ crossing the real axis again.

The branch points are the roots of $p \pm q=0$ :

$$
\lambda_{1}^{*}=-\sqrt{\rho / C_{11}}, \quad \lambda_{2}^{*}=-\sqrt{\rho / C_{66}}, \quad \lambda_{3}^{*}=\sqrt{\rho / C_{11}}, \quad \lambda_{4}^{*}=\sqrt{\rho / C_{66}}
$$

and the roots $\lambda_{5}^{*}, \lambda_{6}^{*}, \lambda_{7}^{*}, \lambda_{8}^{*}$ of $q^{2}=0$ or

$$
\frac{1}{C_{22}^{2} C_{66}^{2}}\left[l_{1} \lambda^{4}+2 \rho l_{2} \lambda^{2}+\rho^{2} l_{3}\right]=0
$$

where

$$
\begin{aligned}
& l_{1}=\left(C_{11} C_{22}-C_{12}^{2}\right)\left[C_{11} C_{22}-\left(C_{12}+2 C_{66}\right)^{2}\right], \\
& l_{2}=\left(C_{12}+C_{66}\right)^{2}\left(C_{22}+C_{66}\right)-\left(C_{11} C_{22}-C_{66}^{2}\right)\left(C_{22}-C_{66}\right), \\
& l_{3}=\left(C_{22}-C_{66}\right)^{2} .
\end{aligned}
$$

According to the values of the elastic coefficients we distinguish the following cases:

(I) $\left(C_{11}-C_{66}\right)\left(C_{22}-C_{66}\right)>\left(C_{66}+C_{12}\right)^{2}$, in which the discriminant of (15) is negative and (15) has complex roots (4 roots symmetrically located with respect to the real and imaginary axes). The four Riemann sheets are attached as shown in Fig. 1a. 

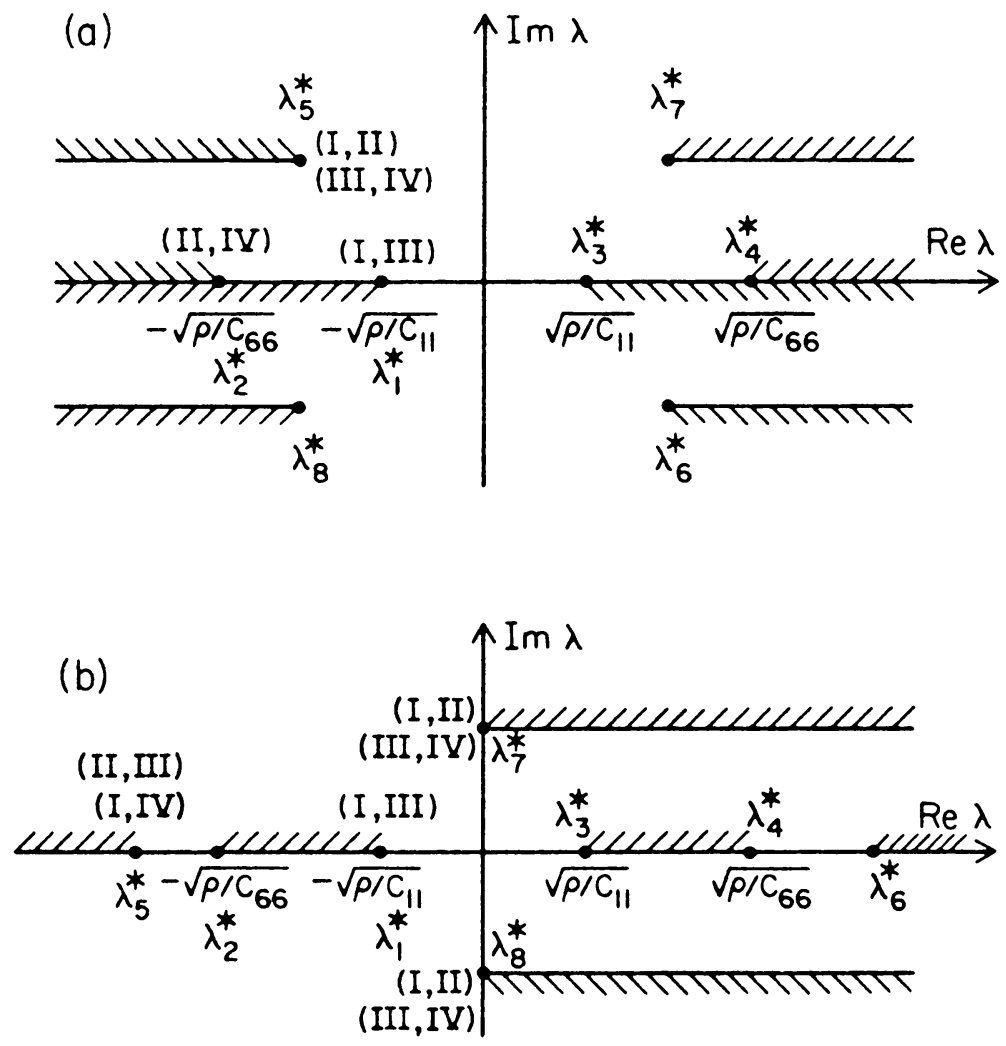

FIG. 1. Branch cuts on the four-sheet Riemann surface (a) Case I, (b) Case II(ii).

(II) $\left(C_{11}-C_{66}\right)\left(C_{22}-C_{66}\right) \leqslant\left(C_{66}+C_{12}\right)^{2}$, in which the discriminant of (15) is positive, so that according to the signs of $l_{1}$ and $l_{2}(15)$ may have either all four roots real, or four pure imaginary, or two real and two pure imaginary.

In this case we distinguish the following two subcases:

(i) $\left(C_{66}+C_{12}\right)^{2}<C_{22}\left(C_{11}-C_{66}\right)$,

(ii) $\left(C_{66}+C_{12}\right)^{2}>C_{22}\left(C_{11}-C_{66}\right)$.

In case II(ii), for any material of cubic symmetry and all material of hexagonal symmetry satisfying the conditions $C_{11}>C_{66}, \sqrt{C_{11}}+\sqrt{C_{11}-C_{66}}>\sqrt{C_{22}}$ (which indeed are satisfied by all hexagonal materials that we could check), equation (15) has two real and two pure imaginary roots. The branch cuts are shown in Fig. $\mathrm{lb}$ and the four Riemann sheets are connected as shown in Fig. $1 \mathrm{~b}$ as well. Zinc belongs to this case.

It is easy to prove that all branch points are of order 1 . Moreover, $\lambda_{5}^{*}, \lambda_{6}^{*}, \lambda_{7}^{*}, \lambda_{8}^{*}$ are double branch points (of order 1). In the isotropic case $\lambda_{5}^{*}, \lambda_{6}^{*}, \lambda_{7}^{*}, \lambda_{8}^{*}$ are no longer branch points since $q(\lambda)$ is a perfect square and the function is not multivalued around that point. 


\section{Inversion of the Laplace transforms.}

1. The Cagniard-de Hoop contour. In order to invert $U_{1}(\lambda)$ and $U_{2}(\lambda)$, we need to integrate the expressions given by (12), i.e.

$$
\hat{u}_{i}\left(x_{1}, x_{2}, s\right)=\frac{s}{2 \pi i} \int_{B r} D_{i}(\lambda) U_{1}(\lambda, 0, s) e^{s \lambda x_{1}-s x_{2} \eta(\lambda)} d \lambda
$$

where the $D_{i}(\lambda)(i=1,2)$ denote the appropriate coefficients in (12), and the integration is carried along the Bromwich contour in both Sheets I and II of the Riemann surface over the complex $\lambda$-plane, which are the only sheets of interest due to the radiation conditions at infinity.

In order to perform the integration in (16) the Bromwich contour is transformed to the Cagniard-de Hoop contour-(in sheets I and II of the Riemann surface)-which is defined by [14]:

$$
\lambda x_{1}-\eta(\lambda) x_{2}=-t, \quad t>0,
$$

where $\eta(\lambda)$ also satisfies (9), i.e.:

$$
\Omega(\lambda, \eta(\lambda))=0
$$

The integral (16) may be written according to the reflection principle as

$$
\frac{s}{\pi} \operatorname{Im} \int_{\Delta^{+}} D_{i}(\lambda) U_{1}(\lambda, 0, s) e^{s \lambda x_{1}-s x_{2} \eta(\lambda)} d \lambda
$$

where $\Delta^{+}$denotes the upper-half parts of the Bromwich contour in Sheets I and II of the Riemann surface.

It is easy to see that the only singularity of the integrand in the upper-half $\operatorname{Re} \lambda<0$ plane is a root of $q=0$, say $\lambda_{5}^{*}$ in Fig. 1a, which is a first-order pole and a branch point of order 1 connecting Sheets I and II. Since the residue of such a first-order pole in a 2-sheet Riemann surface is zero-the Cagniard-de Hoop contour makes two circles around $\lambda_{5}^{*}$, giving no net contribution-there is no contribution due to the pole $\lambda_{5}^{*}$. If the roots of $q=0$ lie on the imaginary axis as in Figure $1 \mathrm{~b}$ then the Bromwich contour would have to go around the pole (twice) but without giving net contribution. Thus the Bromwich contour may be transformed into a Cagniard-de Hoop contour as in Burridge [15]. It can be also proved that the Cagniard-de Hoop contour never meets the branch cuts on the real axis, so there are no contributions due to branch points on the real axis, a fact which should be expected on the physical grounds that there are not head waves due to an edge dislocation in an infinite medium.

We will show now that for Cases I and II(i), the Cagniard-de Hoop contours can never meet the branch cut $\left(-\infty,-\sqrt{\rho / C_{11}}\right)$ on Sheet $I$ and the branch cut $\left(-\infty,-\sqrt{\rho / C_{66}}\right)$ on Sheet II. This is because $\lambda x_{1}-x_{2} \eta(x)$ must be real on the Cagniard-de Hoop contour, but $\eta(x)$ is not real on these segments of the negative $\operatorname{Re} \lambda$ axis, since $p$ and $q$ satisfy 


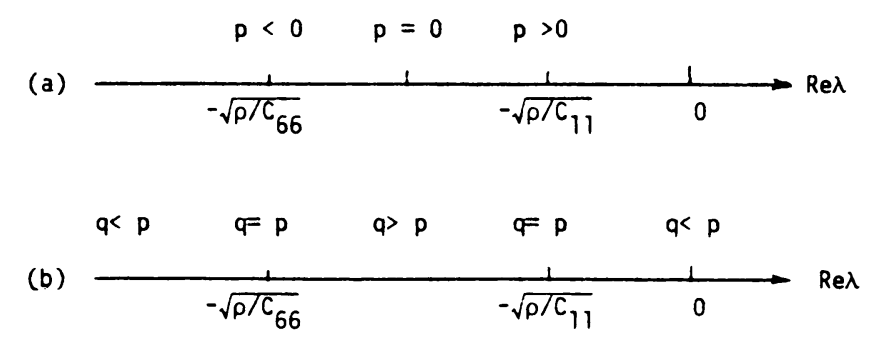

from which it follows:

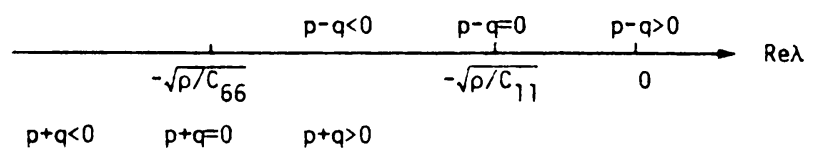

In deriving the ordering shown in (20-1) and (20-2) different cases of relative magnitude of the elastic coefficients were distinguished, as with the investigation of the roots of (15), but the conclusion reached in (20-1) is the same in all cases. From (20-2) it follows that $\eta_{1}=\sqrt{p-q}$ is imaginary in $\left(-\infty,-\sqrt{\rho / C_{11}}\right)$, so that $\lambda x_{1}-\eta_{1} x_{2}$ is imaginary on $(-\infty$, $-\sqrt{\rho / C_{11}}$ ) and the Cagniard-de Hoop contour can never meet this segment. Similarly neither can $\lambda x_{1}-\eta_{2} x_{2}$ meet the segment $\left(-\infty,-\sqrt{\rho / C_{66}}\right)$. By analytic continuation the function $\lambda x_{1}-\eta x_{2}$ cannot even approach the branch cut too closely. Moreover, we see that, for subsonic motion (i.e. $\left.\zeta^{\prime}\left(x_{1}\right)>\sqrt{\rho / C_{66}}>\sqrt{\rho / C_{11}}>0\right), U_{1}(\lambda, 0, s)$ as given by (4-1) does not give a pole inside the Cagniard-de Hoop contour.

For the case II(ii) we have

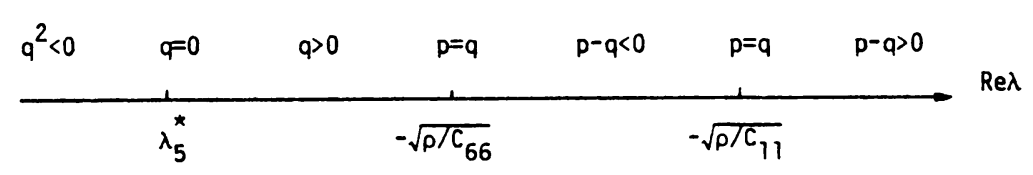

The Cagniard-de Hoop contour will never meet the branch cut $\left(-\infty, \lambda_{5}\right]$ on Sheets I and II since $q$ is imaginary in this interval and hence $\sqrt{p \pm q}$ is complex, and will not meet the branch cut $\left[-\sqrt{\rho / C_{66}},-\sqrt{\rho / C_{11}}\right]$ on Sheet I since $p-q<0$ in this interval.

Then the integral (16) may be transformed into one in the Cagniard-de Hoop contour on two sheets without contribution from branch cuts and branch points. The Cagniard-de Hoop contours may be disjoint on the two sheets or they may be connected. A geometrical interpretation of the Cagniard-de Hoop contour [16], may help in understanding the qualitative behavior of the contour. In the real $\lambda-\eta$ plane, we first plot the slowness surface $\Omega(\lambda, \eta)=0$. We notice that in different cases of material coefficients, the slowness surface has different shapes (e.g. [17]). Typical shapes of slowness surface are shown in Figs. 3 and 2, for Case I and Case II(ii) (zinc) respectively. By physical consideration we are only interested in branches $\eta^{\mathrm{I}}(\lambda)$ and $\eta^{\mathrm{II}}(\lambda)$ of function $\eta(\lambda)$. For Case I the branches $\eta^{\mathrm{I}}(\lambda), \eta^{\mathrm{II}}(\lambda)$ are indicated on Fig. 3a. In Case II(i), the slowness surface is of different type than Case I. But as the correspondence of different branches of $\eta(\lambda)$ to the different parts on the slowness surface is concerned, Case II(i) is similar to Case I. 

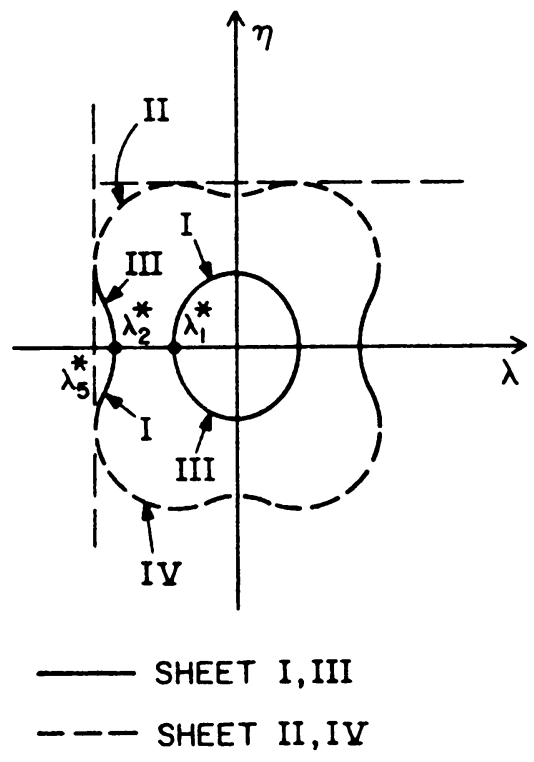

FIg. 2. The surfaces $\eta(\lambda)= \pm \frac{1}{\sqrt{2}} \sqrt{p \pm q}$, for Case II(ii) (Zinc).

However, Case II(ii) is more interesting. In Case II(ii), it is shown that on the slowness surface there exist points with common vertical tangent $\left(\lambda=\lambda_{5}^{*}, \lambda_{6}^{*}\right.$ in Fig. 2), which corresponds to double points of the cusps on the wave front on the $x_{1}$-axis. It is easy to see that in the interval $\left[\lambda_{5}^{*},-\sqrt{\rho / C_{66}}\right], \Omega(\lambda, \eta)=0$ has real solutions both for $\eta=\frac{1}{2} \sqrt{p-q}$ and $\eta=\frac{1}{2} \sqrt{p+q}$. We may easily prove that the point $\lambda_{5}^{*}$ has the maximum value of $|\lambda|$ in the slowness surface.

According to Burridge [13], the branches $\eta^{\mathrm{I}}(\lambda), \eta^{\mathrm{II}}(\lambda)$ correspond to the parts of the slowness surface where the outward normal has a positive $\eta$ component. Hence for Case II(ii), there are parts of the outer sheet of the slowness surface which correspond to $\eta^{\mathrm{I}}(\lambda)$, in other words $\eta^{\mathrm{I}}(\lambda)$ corresponds to several disconnected parts of the slowness surface. This correspondence for Case II(ii) (zinc) is indicated on Fig. 2.

On the same real $\lambda-\eta$ plane we also plot the lines $l_{t}$, parametrised by $t$, which satisfy (17), i.e.

$$
\eta=\lambda \tan \theta+\frac{t}{R \cos \theta}, \quad t>0
$$

where

$$
R^{2}=x_{1}^{2}+x_{2}^{2}, \quad \theta=\tan ^{-1}\left(x_{1} / x_{2}\right) .
$$

The Cagniard-de Hoop contour which is defined by (17) and (18) must satisfy after substitution of (21) into (18):

$$
G(\lambda, t) \equiv \Omega(\lambda, \eta(\lambda, t))=0
$$

which is a 4 th-order equation for $\lambda$, the roots of which correspond obviously to the intersection of the slowness surface with the lines $l_{t}$. As $t$ increases for $R$ and $\theta$ constant, 

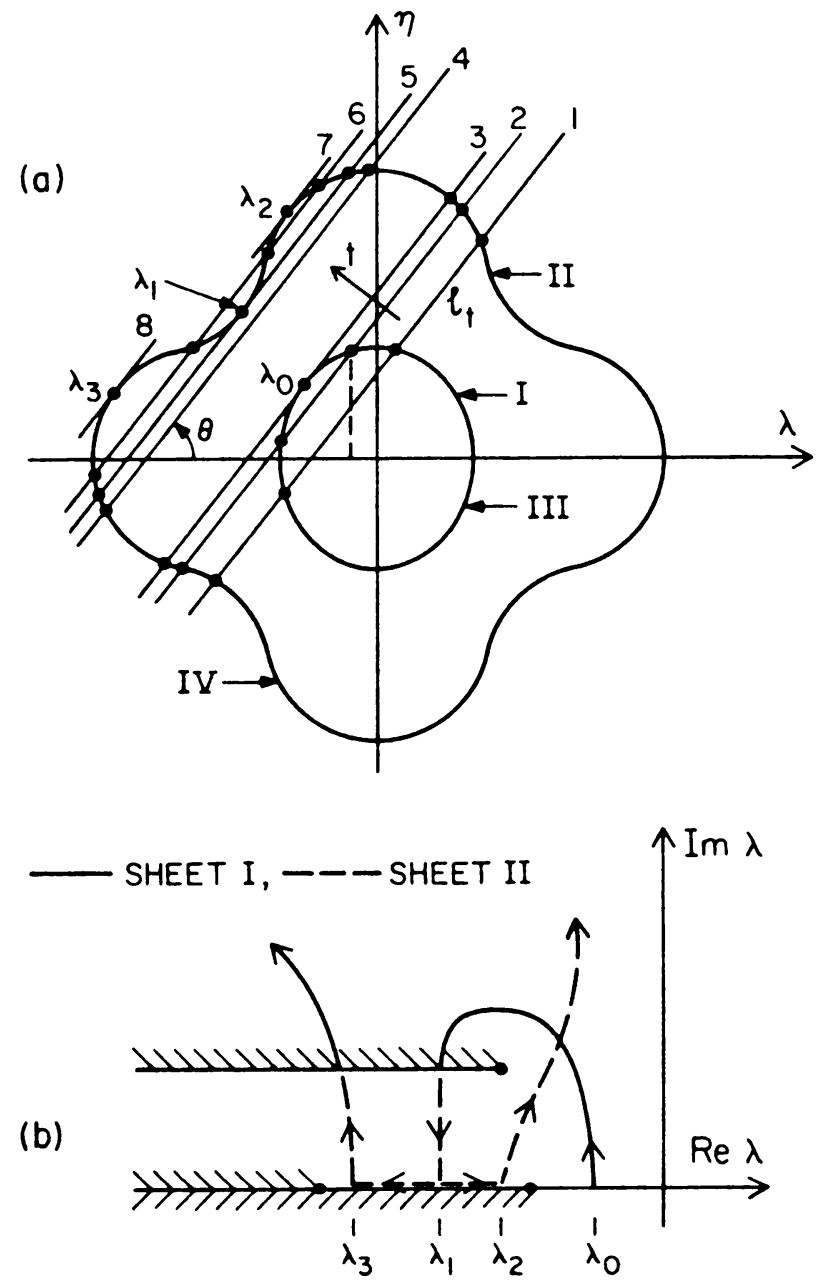

Fig. 3. Schematic of the slowness surface and the Cagniar-de Hoop contour for Case I.

the line $l_{t}$ moves upwards as in Fig. 3. If there are intersections of the line $l_{t}$ with the slowness surface, they correspond to the real part of the Cagniard-de Hoop contour. In position 1 (Fig. 3) there are 4 real roots ( 2 in Sheets I and II, 2 in Sheets II and IV (of no interest)). In position 2 there are 4 real roots ( 2 in Sheet I, 1 in Sheet II, and 1 in Sheet IV). In position 3 there are 1 repeated real root, 1 real root in Sheet II and 1 in Sheet IV. The point $\lambda_{0}$ (in position 3 with corresponding time $t_{0}$ ) is a bifurcation point of the family of solutions, which splits for $t>t_{0}$ to two complex roots. Thus in position 4 there are 2 complex roots for Sheet I, and these two are continuous (by the implicit function theorem, since $G(\lambda, t)$ is continuous) as $t$ varies from position 3 to 5 , i.e. from Sheet I to Sheet II. This means that the Cagniard-de Hoop contour in Sheet I is connected to the Cagniard-de Hoop contour in Sheet II and the transition from Sheet I to Sheet II occurs at some time between $t_{3}$ and $t_{5}$. At the position 5 we have two real roots and 1 repeated real root. This 

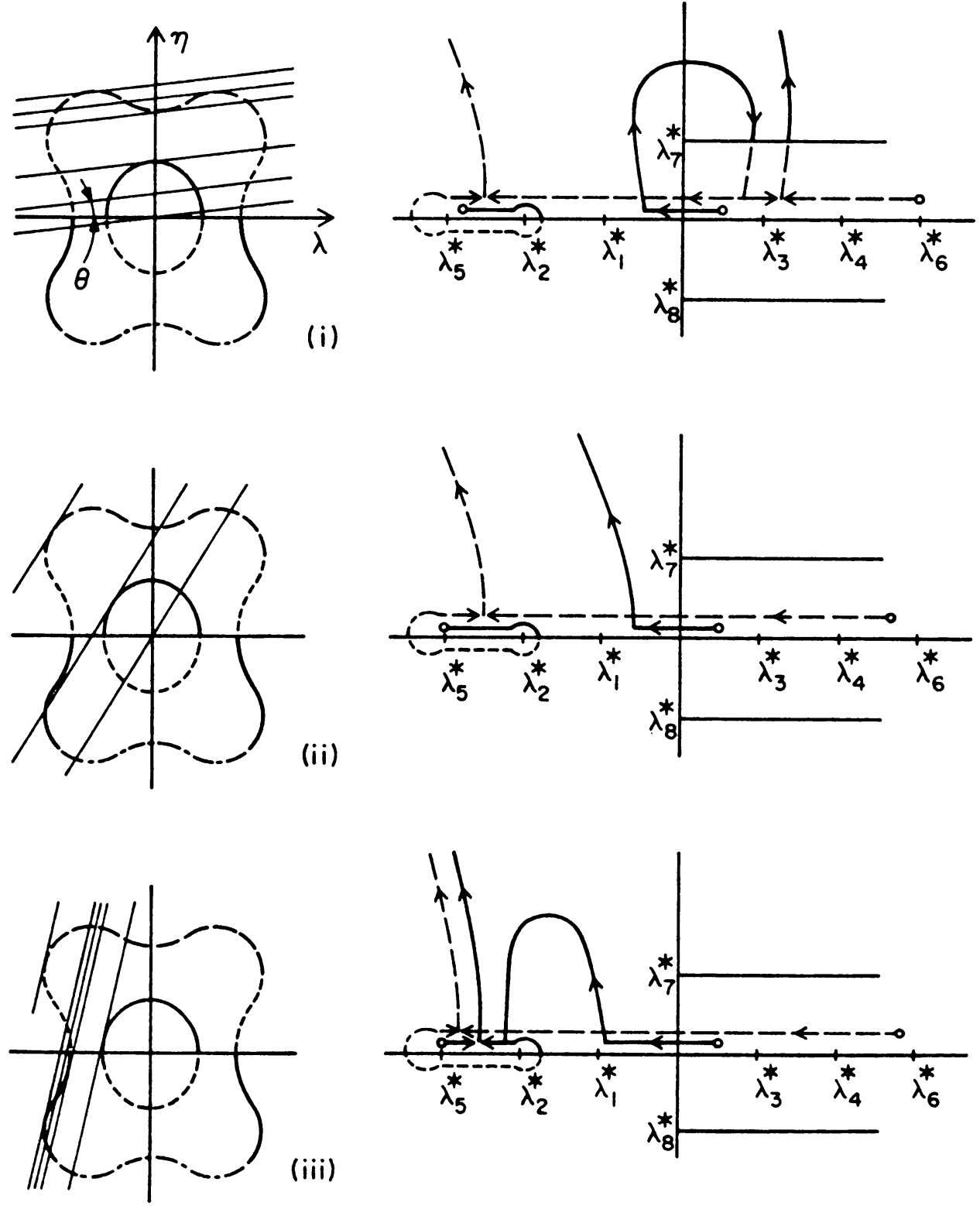

(ii)

SHEET I , - - SHEET II, -----SHEET III. - - - - SHEET IV

FIG. 4. Schematic of the slowness surface and the Cagniard-de Hoop contour for Case II(ii) (Zinc).

is a bifurcation point, in which the two complex roots in Sheet II become a repeated real, to split into 2 real roots in position 6. Thus the part of the Cagniard-de Hoop contour corresponding between positions 5 and 7 is along the real $\lambda$-axis. Two real roots coalesce in a repeated root at 7, which then bifurcates into 2 complex roots. At position 8 the remaining two roots coalesce into a repeated root. The physical meaning of the bifurcation 
points will be discussed in the next section regarding the investigation of the wave-front behavior. In the case of zinc (II(ii)) the Cagniard-de Hoop contour will have either one of the three forms indicated in Fig. 4 according to the slope of the lines $\eta=\lambda \tan \theta+$ $t / R \cos \theta$. Here we have only considered materials in which the inner sheet of the slowness surface does not intersect the outer sheet, that is we do not consider singular hyperbolic cases [8] which constitute the object of part II of this research.

2. Solution for constant and general velocity dislocation motion. Let us assume an edge dislocation starting from rest at time $t=0$ and moving with constant velocity $x_{1}=v_{d} t$ or equivalently $t=\alpha x_{1}\left(\alpha=1 / v_{d}\right)$, with $v_{d}<\sqrt{C_{66} / \rho}<\sqrt{C_{11} / \rho}$ for subsonic motion.

Equation (4-1) may be integrated explicitly to give

$$
v_{1}(\lambda, 0, s)=\frac{-\Delta u}{2 s} \int_{0}^{\infty} e^{-s \alpha \xi-s \lambda \xi} d \xi=-\frac{\Delta u}{2 s} \frac{1}{s(\alpha+\lambda)}
$$

so that for the strain component $\partial \hat{u}_{i} / \partial x_{2}$, we have from (19)

$$
\frac{\partial \hat{u}_{i}}{\partial x_{2}}\left(x_{1}, x_{2}, s\right)=\frac{\Delta u}{2 \pi} \operatorname{Im} \int_{\Delta^{+}} \frac{D_{i}(\lambda) \eta(\lambda)}{\alpha+\lambda} e^{s \lambda x_{1}-s \eta(\lambda) x_{2}} d \lambda
$$

which with the Cagniard-de Hoop transformation (17) yields:

$$
\frac{\partial \hat{u}_{i}}{\partial x}\left(x_{1}, x_{2}, s\right)=\left.\frac{\Delta u}{2 \pi} \operatorname{Im} \int_{C^{+}}\left\{\frac{D_{i}(\lambda) \eta(\lambda)}{\alpha+\lambda} \frac{d \lambda}{d t}\right\}\right|_{\lambda=\lambda\left(t, x_{1}, x_{2}\right)} e^{-t s} d \tau
$$

where $C^{+}$is the upper part (i.e. $\operatorname{Im} \lambda \geqslant 0$ ) of the Cagniard-de Hoop contour and $\lambda=\lambda\left(t, x_{1}, x_{2}\right)$ is a solution of (21) and (23).

Then as in $[15,16]$, the inversion of the Laplace transform can be written as

$$
\frac{\partial u_{i}}{\partial x_{2}}\left(x_{1}, x_{2}, t\right)=\left.\frac{\Delta u}{2 \pi} \sum_{\lambda\left(t, x_{1}, x_{2}\right) \in C^{+}} \operatorname{Im}\left\{\frac{D_{i}(\lambda) \eta(\lambda)}{\alpha+\lambda} \frac{d \lambda}{d t}\right\}\right|_{\lambda=\lambda\left(t, x_{1}, x_{2}\right)} H\left(t-t_{N}\right)
$$

where $t_{N}(N=1,2)$ is the value at which the line $l_{t_{N}}$ defined in the equation (21) contains the point $\lambda=0$ on the sheet $N$ of the slowness surface. In fact, the above expression for $\partial u_{i} / \partial x_{2}$ can be evaluated analytically.

Consider now an edge dislocation starting from rest and moving nonuniformly according to $x_{1}=l(t)$ or equivalently $t=\zeta\left(x_{1}\right)$, and let us furthermore assume that the motion is subsonic, i.e.

$$
\zeta^{\prime}\left(x_{1}\right)>\sqrt{\rho / C_{66}}>\sqrt{\rho / C_{11}}>0 .
$$

In this case the integral $\int_{0}^{\infty} e^{-s \xi(\xi)-s \lambda \xi} d \xi$ cannot be evaluated explicitly. Then for the strain component $\partial \hat{u}_{i} / \partial x_{2}$ we have from (19)

$$
\frac{\partial \hat{u}_{i}}{\partial x_{2}}\left(x_{1}, x_{2}, s\right)=\frac{s \Delta u}{2 \pi} \operatorname{Im} \int_{\Delta^{+}} \int_{0}^{\infty} D_{i}(\lambda) \eta(\lambda) e^{s \lambda\left(x_{1}-\xi\right)-s \eta(\lambda) x_{2}-s \xi(\xi)} d \xi d \lambda .
$$

By similar consideration as in Markenscoff [18] the interchange in integration in $\lambda$ and $\xi$ is permissible for $x_{2} \neq 0$. Then for $x_{2} \neq 0$ we have

$$
\frac{\partial \hat{u}_{i}}{\partial x_{2}}\left(x_{1}, x_{2}, s\right)=\frac{s \Delta u}{2 \pi} \operatorname{Im} \int_{0}^{\infty} e^{-s \xi(\xi)} \int_{\Delta^{+}} D_{i}(\lambda) \eta(\lambda) e^{s \lambda\left(x_{1}-\xi\right)-s \eta(\lambda) x_{2}} d \lambda d \xi
$$


and we can proceed in evaluating the innermost integral by the Cagniard-de Hoop technique as described in the constant velocity case. For each fixed $\xi \in[0, \infty)$ the corresponding Cagniard-de Hoop contour $C(\xi)$ is defined by equations (21) and (23) in which now

$$
R^{2}(\xi)=(x-\xi)^{2}+x_{2}^{2}, \theta(\xi)=\tan ^{-1}\left[\left(x_{1}-\xi\right) / x_{2}\right],
$$

so that we have

$$
\frac{\partial \hat{u}_{i}}{\partial x_{2}}\left(x_{1}, x_{2}, s\right)=\left.\frac{s \Delta u}{2 \pi} \int_{0}^{\infty} e^{-s \zeta(\xi)} \operatorname{Im} \int_{C^{+}(\xi)}\left\{D_{i}(\lambda) \eta(\lambda) \frac{d \lambda}{d t}\right\}\right|_{\lambda=\lambda\left(t, x_{1}-\xi, x_{2}\right)} e^{-t s} d t .
$$

Hence

$$
\begin{array}{r}
\frac{\partial u_{i}}{\partial x_{2}}\left(x_{1}, x_{2}, t\right) \\
=\left.\frac{\Delta u}{2 \pi} \frac{\partial}{\partial t} \int_{0}^{\infty} \sum_{\lambda\left(t-\zeta(\xi), x_{1}-\xi, x_{2}\right) \in C^{+}(\xi)} \operatorname{Im}\left\{D_{i}(\lambda) \eta(\lambda) \frac{d \lambda}{d t}\right\}\right|_{\lambda=\lambda\left(t-\xi(\xi), x_{1}-\xi, x_{2}\right)} \\
\cdot H\left(t-t_{N}(\xi)-\zeta(\xi)\right) d \xi
\end{array}
$$

where $t_{N}(\xi)$ is the value at which the corresponding $l_{t_{N(\xi)}}$ defined in (21) for $R(\xi), \theta(\xi)$ contains the point $\lambda=0$ on the sheet $N$ of the slowness surface.

\section{Asymptotic wavefront-analysis by the method of steepest descent.}

1. Saddle-points on the Cagniard-de Hoop contour. In order to obtain the wavefront behavior explicitly for general velocity dislocation motion we proceed with the asymptotic evaluation of the integral (16) as $s \rightarrow \pm \infty$ [19] and use the method of steepest descent [20].

We need to find the critical points which are the saddle points of the exponent $F(\lambda) \equiv \lambda x_{1}-\eta(\lambda) x_{2}$, determine their order, and evaluate the integral on a path of steepest descent [20].

It is easy to see that the Cagniard-de Hoop contour satisfying the definition $F(\lambda)=-t$ or equivalently $\operatorname{Im} F(\lambda)=0$ is a curve of steepest descent as $t$ increases [20, p. 255].

The first-order saddle-points satisfy

$$
\frac{d F(\lambda)}{d \lambda}=x_{1}-x_{2} \eta^{\prime}(\lambda)=0, \quad \Omega(\lambda, \eta(\lambda))=0, \quad \frac{d^{2} F}{d \lambda^{2}} \neq 0
$$

or equivalently

$$
\left.\frac{d \Omega}{d \lambda}(\lambda, \eta(\lambda, t))\right|_{\eta^{\prime}=\tan \theta}=0, \quad \Omega(\lambda, \eta)=0,\left.\quad \frac{d^{2} \Omega}{d \lambda^{2}}(\lambda, \eta)\right|_{\eta^{\prime}=\tan \theta} \neq 0 .
$$

First-order saddle points on the Cagniard-de Hoop contour must satisfy equations (27) together with (21), i.e.

$$
G(\lambda, t)=0, \quad \frac{\partial G}{\partial \lambda}(\lambda, t)=0, \quad \frac{\partial^{2} G}{\partial \lambda^{2}} \neq 0
$$

where $G(\lambda, t)$ was defined in (23).

In order to find the saddle-points (first-order) we need to solve equations (28) simultaneously, which corresponds to finding the repeated root of $G(\lambda, t)=0$. From (23): 


$$
G(\lambda, t)=a \lambda^{4}+4 b \lambda^{3}+6 c \lambda^{2}+4 d \lambda+e=0
$$

where

$$
\begin{aligned}
& a=\sin ^{4} \theta+e_{1} \sin ^{2} \theta \cos ^{2} \theta+e_{4} \cos ^{4} \theta, \\
& b=\frac{t}{2 R} \sin \theta\left(2 \sin ^{2} \theta+e_{1} \cos ^{2} \theta\right), \\
& c=\frac{1}{6}\left[\left(6 \sin ^{2} \theta+e_{1} \cos ^{2} \theta\right)\left(\frac{t}{R}\right)^{2}-\left(e_{3} \sin ^{2} \theta+e_{2} \cos ^{2} \theta\right) \rho \cos ^{2} \theta\right] \\
& d=\frac{t}{2 R} \sin \theta\left(2\left(\frac{t}{R}\right)^{2}-\rho e_{3} \cos ^{2} \theta\right), \\
& e=\left(\frac{t}{R}\right)^{4}-\rho e_{3} \cos ^{2} \theta\left(\frac{t}{R}\right)^{2}+\frac{\rho^{2}}{C_{22} C_{66}} \cos ^{4} \theta
\end{aligned}
$$

and

$$
e_{1}=\frac{C_{11} C_{22}-C_{12}^{2}-2 C_{12} C_{66}}{C_{22} C_{66}}, \quad e_{2}=\frac{C_{11}+C_{66}}{C_{22} C_{66}}, \quad e_{3}=\frac{C_{22}+C_{66}}{C_{22} C_{66}}, e_{4}=\frac{C_{11}}{C_{22}} .
$$

The equation $G(\lambda, t)=0$ has a repeated root [21, p. 205] iff the discriminant

$$
D \equiv 256\left(I^{3}-27 J^{2}\right)=0
$$

where

$$
I=a e-4 b d+3 c^{2} \quad \text { and } \quad J=\operatorname{det}\left|\begin{array}{lll}
a & b & c \\
b & c & d \\
c & d & e
\end{array}\right|,
$$

with the reducing cubic being

$$
4 a^{3} \lambda^{3}-a I \lambda+J=0 .
$$

Equation (30) is a 6 th order equation for $t^{2}$ and we are interested only in the positive roots of it.

We can solve (30) numerically for a maximum of 6 positive roots $t_{i}(i=0,1, \ldots, 5)$.

A second-order saddle-point must satisfy

$$
G(\lambda, t)=0, \quad G,_{\lambda}(\lambda, t)=0, \quad G,_{\lambda \lambda}(\lambda, t)=0, \quad G,_{\lambda \lambda \lambda} \neq 0
$$

the solution of which is the 3-fold root of $G(\lambda, t)=0$. We can prove that (32) can have a solution only for a finite number of angles $\theta$. These points correspond to the tips of the cusps of the wave surface (for conditions of existence of which see e.g. [17]). We were not able to prove that there do not exist 3rd-order saddle points on the Cagniard-de Hoop contour. We can also see that at most for a finite number of angles $\theta$, there may exist complex first-order saddle-oints on the Cagniard-de Hoop contour. However, a complex first-order saddle-point would imply 4 complex roots of Eq. (23), or 4 complex intersections of line $l_{t}$ with the slowness surface. This can happen only for time $t>t_{8}$, i.e. after the wavefront has passed. Moreover there do not exist second-order complex saddle-points since that 3-fold root of $G(\lambda, t)=0$ cannot be complex.

For a real first-order saddle-point $\lambda_{0}$, if $d^{2} F\left(\lambda_{0}\right) / d \lambda^{2}=a e^{i \alpha}, a>0$, then the directions of steepest descent from $\lambda_{0}$ are [20, p. 255]: 


$$
-\frac{\alpha}{2}+\frac{\pi}{2}, \quad-\frac{\alpha}{2}+\frac{3 \pi}{2}
$$

and the directions of steepest ascent

$$
-\frac{\alpha}{2}, \quad-\frac{\alpha}{2}+\pi
$$

For $d^{2} F\left(\lambda_{0}\right) / d \lambda^{2}>0, \alpha=0$ and the direction of steepest descent is normal to the real $\lambda$-axis; that is, $\lambda_{0}$ corresponds to a maximum of $F(\lambda)$ along the complex part of the Cagniard-de Hoop contour. For $d^{2} F\left(\lambda_{0}\right) / d \lambda^{2}<0, \alpha=\pi$ and the direction of steepest ascent is normal to the real $\lambda$-axis, that is $\lambda_{0}$ corresponds to a minimum of $F(\lambda)$ along the complex part of the Cagniard-de Hoop contour. In the isotropic case

$$
F^{\prime \prime}\left(\lambda_{0}\right)=x_{2} \frac{\rho \sqrt{C_{66}}}{\left(\rho-C_{66} \lambda^{2}\right)^{3 / 2}}>0,
$$

so that all saddle-points are maximum points along the complex part of the Cagniard-de Hoop contour.

For a fixed point in space, $P$, that is for $R$ and $\theta$ fixed, we find up to a maximum of six real solutions $\left(\lambda_{0}, t_{0}\right), \ldots,\left(\lambda_{5}, t_{5}\right)$ to Eq. (28) or (32). Equivalently to (28) or (32), $\left(\lambda_{0}, t_{0}\right), \ldots,\left(\lambda_{5}, t_{5}\right)$ satisfy

$$
\begin{aligned}
\Omega(\lambda, \eta) & =0, \\
x_{1} & =-\Omega_{\lambda}^{\prime} x_{2} / \Omega_{\eta}^{\prime}, \\
t_{0,1, \ldots .5} & =-\lambda_{0,1, \ldots .5}\left(-\Omega_{\lambda}^{\prime} x_{2} / \Omega_{\eta}^{\prime}\right)+\eta x_{2},
\end{aligned}
$$

which can be written as:

$$
\begin{aligned}
x_{1} & =\frac{-t \Omega_{\lambda}^{\prime}}{\lambda \Omega_{\lambda}^{\prime}+\eta \Omega_{\eta}^{\prime}}, \\
x_{2} & =\frac{t \Omega_{\eta}^{\prime}}{\lambda \Omega_{\lambda}^{\prime}+\eta \Omega_{\eta}^{\prime}}, \\
\Omega(\lambda, \eta) & =0 .
\end{aligned}
$$

Equations (33) for any fixed time $t$, represent the wave-surface [16], so that the times $t_{0}, \ldots, t_{5}$ correspond to the wavefront passing from the point $P$, and the asymptotic approximation of the integral around the saddle-point represents the wavefront behavior.

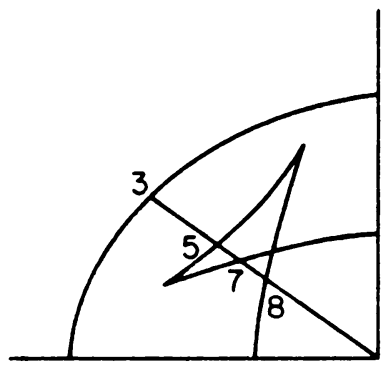

FIG. 5. Schematic of the wave-surface for Case I. 
Maximum saddle-points correspond to initiation of motion while minimum saddle-points correspond to termination of motion [22].

If we refer to Fig. 3, the saddle point at the position 3 is a maximum and corresponds to the outer wavefront on Fig. 5. The saddle-point at the position 5 is a minimum and corresponds to the termination of a given motion, since the transition from Sheet I to Sheet II has begun at an intermediate position 4. At positions 7 and 8 the maximum saddle-points correspond to the initiation of motion. In the region inside the cuspidal area there is no motion, since between points 5 and 7 the Cagniard-de Hoop contour lies along the real axis and there is no contribution to the integral (19).

2. Asymptotic evaluation of the integral for constant velocity dislocation motion (subsonic). We proceed now with the asymptotic evaluation of the integral in (24) about the saddle points that were found in the previous section.

Let $\lambda_{0}, \ldots, \lambda_{5}$ be the saddle-points corresponding to the solutions $t_{j}$ of Eq. (30) according to $F\left(\lambda_{j}\right)=-t_{j}$. We divide then the upper part (i.e. $\operatorname{Im} \lambda \geqslant 0$ ) of the Cagniard-de Hoop contour into $\Sigma_{j=0}^{5} C\left(\lambda_{j}\right)$ with each $C\left(\lambda_{j}\right)$ including one saddle-point, and we have from (24)

$$
\frac{\partial \hat{u} i}{\partial x_{2}}\left(x_{1}, x_{2}, s\right)=\frac{\Delta u}{2 \pi} \sum_{j=0}^{b} \operatorname{Im} \int_{C\left(\lambda_{j}\right)} \frac{D_{i}(\lambda) \eta(\lambda)}{\alpha+\lambda} e^{s \lambda x_{1}-s \eta(\lambda) x_{2}} d \lambda .
$$

Evaluating asymptotically [20] the integral (34) around the saddle-point $\lambda_{j}$ assumed a maximum on the complex Cagniard-de Hoop contour, we obtain, as an example, for the strain component $\partial \hat{u}_{1} / \partial x_{2}$

$$
\begin{aligned}
\frac{\partial \hat{u}_{1}}{\partial x_{2}}\left(x_{1}, x_{2}, s\right) & =\operatorname{Im} \int_{C\left(\lambda_{j}\right)} \frac{\Delta u}{2 \sqrt{2} \pi}\left[E^{ \pm}(\lambda) /(\alpha+\lambda)\right] e^{s \lambda x-s x_{2} \sqrt{p^{ \pm q}} / \sqrt{2}} d \lambda \\
& \sim \frac{\Delta u}{4 \sqrt{\pi}} \frac{e^{s F\left(\lambda_{j}\right)} E_{1}^{ \pm}\left(\lambda_{j}\right)}{s^{1 / 2} F^{\prime \prime}\left(\lambda_{j}\right)^{1 / 2}\left(\alpha+\lambda_{j}\right)} \text { as } s \rightarrow \infty
\end{aligned}
$$

with $E_{1}^{ \pm}(\lambda)=D_{1}^{ \pm}(\lambda) \sqrt{p \pm q}$ and the \pm signs corresponding to whether $\lambda_{j}$ belongs to Sheet II or Sheet I respectively.

In order to invert the Laplace transform in time we define the function

$$
\hat{\omega}\left(x_{1}, x_{2}, s\right) \equiv e^{-S F\left(\lambda_{j}\right)} \frac{\partial \hat{u}_{1}}{\partial x_{2}}\left(x_{1}, x_{2}, s\right) \quad \text { as } s \rightarrow \infty
$$

so that

$$
\frac{\partial u_{1}}{\partial x_{2}}\left(x_{1}, x_{2}, t\right)=\omega\left(t+F\left(\lambda_{j}\right)\right) H\left(t+F\left(\lambda_{j}\right)\right),
$$

and invoke for the inversion of $\hat{\omega}$, as given by (35), a Tauberian theorem [23, p. 192] which, under some restrictions of monotonicity of $\omega\left(x_{1}, x_{2}, t\right)$, gives as $t \rightarrow 0^{+}$

$$
\omega\left(x_{1}, x_{2}, t\right) \sim \frac{\Delta u}{4 \sqrt{\pi}} \frac{t^{-1 / 2} F^{\prime \prime}\left(\lambda_{j}\right)^{-1 / 2} E_{1}^{ \pm}\left(\lambda_{j}\right)}{\Gamma(1 / 2)\left(\alpha+\lambda_{j}\right)}
$$


from which and (36), it follows that

$$
\frac{\partial u_{1}}{\partial x_{2}}\left(x_{1}, x_{2}, t\right) \sim \frac{\Delta u}{4 \pi} \frac{H\left(t-t_{j}\right) E_{1}^{ \pm}\left(\lambda_{j}\right)}{F^{\prime \prime}\left(\lambda_{j}\right)^{1 / 2} \sqrt{t-t_{j}}\left(\alpha+\lambda_{j}\right)} \quad \text { as } t \rightarrow t_{j} .
$$

For a saddle point $\lambda_{k}$ that corresponds to a minimum along the complex Cagniard-de Hoop contour, we proceed as follows. We decompose $\partial u_{1}\left(x_{1}, x_{2}, t\right) / \partial x_{2}$ into two parts as

$$
\frac{\partial u_{1}}{\partial x_{2}}\left(x_{1}, x_{2}, t\right)=\frac{\partial u_{1}}{\partial x_{2}}\left(x_{1}, x_{2}, t\right) H\left(t_{k}-t\right)+\frac{\partial u_{1}}{\partial x_{2}}\left(x_{1}, x_{2}, t\right) H\left(t-t_{k}\right), \quad t \geqslant 0,
$$

so that

$$
\begin{aligned}
\frac{\partial \hat{u}_{1}}{\partial x_{2}}\left(x_{1}, x_{2}, s\right)= & \int_{0}^{\infty} e^{-s t} \frac{\partial u_{1}}{\partial x_{2}}\left(x_{1}, x_{2}, t\right) H\left(t_{k}-t\right) d t \\
& +\int_{0}^{\infty} e^{-s t} \frac{\partial u_{1}}{\partial x_{2}}\left(x_{1}, x_{2}, t\right) H\left(t-t_{k}\right) d t .
\end{aligned}
$$

The first integral in (38) may be written:

$$
\begin{aligned}
& \int_{0}^{t_{k}} e^{-s t} \frac{\partial u_{1}}{\partial x_{2}}\left(x_{1}, x_{2}, t\right) H\left(t_{k}-t\right) d t \\
= & \int_{-t_{k}}^{0} e^{-s\left(\tau+t_{k}\right)} \frac{\partial u_{1}}{\partial x_{2}}\left(x_{1}, x_{2}, \tau+t_{k}\right) H(-\tau) d \tau \\
= & e^{-s t_{k}} \int_{0}^{\infty} e^{-\hat{p} u} \frac{\partial u_{1}}{\partial x_{2}}\left(x_{1}, x_{2}, t_{k}-u\right) H(u) d u
\end{aligned}
$$

where $\hat{p} \equiv-s$ and use was made of the fact that $\partial u_{1}\left(x_{1}, x_{2}, t\right) / \partial x_{2}=0$ for $t<0$.

Since $\lambda_{k}$ corresponds to a minimum of $F(\lambda)$ along $C\left(\lambda_{k}\right)$, we have $t=-F(\lambda)<$ $-F\left(\lambda_{k}\right)=t_{k}$ so that the integral along $C\left(\lambda_{k}\right)$ in (34) is just as the first integral in (38). Hence as $\hat{p}=-s \rightarrow \infty$, we have

$$
\begin{aligned}
\int_{0}^{\infty} e^{-\hat{p} u} \frac{\partial u_{1}}{\partial x_{2}}\left(x_{1}, x_{2}, t-u\right) d u & =\frac{\partial \hat{u}_{1}}{\partial x_{2}}\left(x_{1}, x_{2},-\hat{p}\right) \\
& \sim \frac{\Delta u e^{-\hat{p} F\left(\lambda_{k}\right)} E_{1}^{ \pm}\left(\lambda_{k}\right)}{4 \sqrt{\pi} \hat{p}^{1 / 2}\left|F^{\prime \prime}\left(\lambda_{k}\right)\right|^{1 / 2}\left(\alpha+\lambda_{k}\right)} .
\end{aligned}
$$

Proceeding to invert (40) in a way analogous to (39), we obtain as $u \rightarrow 0$, or equivalently as $t \rightarrow t_{k}^{-}$:

$$
\frac{\partial u_{1}}{\partial x_{2}}\left(x_{1}, x_{2}, t\right) \sim \frac{\Delta u\left|F^{\prime \prime}\left(\lambda_{k}\right)\right|^{-1 / 2} H\left(t_{k}-t\right) E_{1}^{ \pm}\left(\lambda_{k}\right)}{4 \pi\left(\alpha+\lambda_{k}\right) \sqrt{t_{k}-t}} .
$$

Next we evaluate the solution in the neighborhood of 2nd-order saddle-points $\lambda_{l}$ corresponding to inflection points on the slowness surface (Fig. 3), and to the vertex (tip) of the cusp on the wave-surface (Fig. 5).

From a second-order saddle-point the directions of steepest descent for $F^{\prime \prime}\left(\lambda_{0}\right)>0$, are $\pi / 3, \pi$ and $5 / 3 \pi$, and those of steepest ascent $0,2 \pi / 3,4 \pi / 3$, and vice-versa for $F^{\prime \prime}\left(\lambda_{0}\right)<0$. 


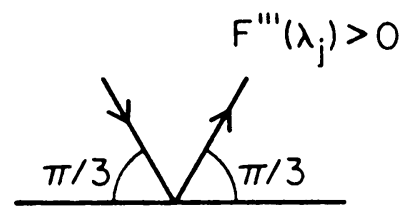

(a)

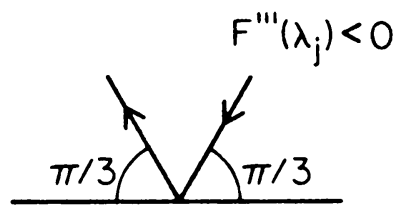

(b)

FIG. 6. Second-order saddle points on the Cagniard-de Hoop contour.

The Cagniard-de Hoop contour near a second-order saddle-point $\lambda_{l}$ is shown in Figs. 6(a) and $6\left(\right.$ b) for $F^{\prime \prime}\left(\lambda_{l}\right)>0$ and $F^{\prime \prime}\left(\lambda_{l}\right)<0$ respectively.

Evaluation of $\partial \hat{u}_{1}\left(x_{1}, x_{2}, s\right) / \partial x_{2}$ as $s \rightarrow \infty$ along the contour of Fig. 6(a) gives

$$
\frac{\partial \hat{u}_{1}}{\partial x}\left(x_{1}, x_{2}, s\right) \sim \frac{\sqrt{3} \Delta u E_{1}^{+}\left(\lambda_{l}\right) e^{s F\left(\lambda_{l}\right)}}{4 \sqrt{2} \pi\left(\alpha+\lambda_{l}\right)}\left(\frac{6}{s F^{\prime \prime}\left(\lambda_{l}\right)}\right)^{1 / 3} \int_{0}^{\infty} e^{-u^{3}} d u
$$

which inverted in time yields as $t \rightarrow\left(-F\left(\lambda_{l}\right)\right)^{+}$

$$
\frac{\partial u_{1}}{\partial x_{2}}\left(x_{1}, x_{2}, t\right) \sim \frac{\Delta u E_{1}^{+}\left(\lambda_{l}\right)}{4 \sqrt{6} \pi\left(\alpha+\lambda_{l}\right)}\left[\frac{6}{F^{\prime \prime}\left(\lambda_{l}\right)}\right]^{1 / 3} \frac{H\left(t+F\left(\lambda_{l}\right)\right)}{\left(t+F\left(\lambda_{l}\right)\right)^{2 / 3}} .
$$

Evaluation of $\partial \hat{u}_{1}\left(x_{1}, x_{2}, s\right) / \partial x_{2}$ as $s \rightarrow-\infty$ along the contour of Fig. 6(b) gives

$$
\frac{\partial \hat{u}_{1}}{\partial x_{2}}\left(x_{1}, x_{2}, s\right) \sim \frac{\sqrt{3} \Delta u E_{1}^{+}\left(\lambda_{l}\right) e^{s F\left(\lambda_{l}\right)}}{4 \sqrt{2} \pi} \int_{0}^{\infty} e^{-u^{3}} d u\left(\frac{6}{s F^{\prime \prime}\left(\lambda_{l}\right)}\right)^{1 / 3}
$$

and

$$
\begin{aligned}
\frac{\partial u_{1}}{\partial x_{2}}\left(x_{1}, x_{2}, t\right) \sim \frac{\Delta u E_{1}^{+}\left(\lambda_{l}\right)}{4 \sqrt{6} \pi\left(\alpha+\lambda_{l}\right)}\left[\frac{6}{F^{\prime \prime}\left(\lambda_{l}\right)}\right]^{1 / 3} \frac{H\left(t_{l}-t\right)}{\left(t_{l}-t\right)^{2 / 3}} \\
\text { as } t \rightarrow t_{l}^{-},\left(t_{l}=-F\left(\lambda_{l}\right)\right) .
\end{aligned}
$$

Thus (42) and (43) give the same result from either direction that we may approach the tip of the cusp. The stress field is more singular ( $2 / 3$ singularity) near these points.

3. Asymptotic evaluation of the integral for general nonuniform motion (subsonic). In the general nonuniform velocity dislocation motion (see Sec. II), we need to evaluate asymptotically the double integral (26).

For a double integral-see e.g. Sirovich $[24$, p. 136] - the main contribution comes from the neighborhood of the point $\left(\lambda_{*}, \xi_{*}\right)$ that satisfies

$$
\partial h / \partial \lambda=0, \quad \partial h / \partial \xi=0
$$

where $h(\lambda, \xi) \equiv \lambda x_{1}-\lambda \xi-x_{2} \eta(\lambda)-\zeta(\xi)$, which are equivalent to

$$
\begin{aligned}
\left(x_{1}-\xi\right)-x_{2} \eta^{\prime}(\lambda) & =0, \\
\lambda+\zeta^{\prime}(\xi) & =0 .
\end{aligned}
$$


But in view of the condition (25) we have that $-\operatorname{Re}\left(\lambda+\zeta^{\prime}(\xi)\right)<0$, so that the maximum of the integrand in (26) occurs at the boundary $\xi=0$, and the main contribution to the integral comes from the neighborhood of the point $\left(\lambda_{*}, \xi\right)=\left(\lambda_{*}, 0\right)$, such that

$$
\partial h(\lambda, 0) /\left.\partial \lambda\right|_{\lambda=\lambda_{*}}=0
$$

the solutions to which have been analyzed in Section IV-1 of this paper.

According to Sirovich [24], we proceed to expand the integrand about the point $\left(\lambda_{*}, 0\right)$ and integrate. Let us assume $l(t)=0(t)$ as $t \rightarrow 0$, or $\zeta^{\prime}(\xi) \rightarrow \infty$ as $\xi \rightarrow 0$.

Then

$$
\begin{aligned}
\int_{0}^{\infty} e^{-s(\lambda \xi+\zeta(\xi))} d \xi & =\int_{0}^{\infty} e^{-s(\lambda(t)+t)} l^{\prime}(t) d t \\
& =\frac{1}{\lambda} \int_{0}^{\infty} e^{-s(\lambda l(t)+t)}\left[\lambda l^{\prime}(t)+1\right] d t-\frac{1}{\lambda} \int_{0}^{\infty} e^{-s(\lambda l(t)+t)} d t \\
& =\frac{1}{\lambda} \int_{0}^{\infty} e^{-s t}\left[1-e^{-s \lambda l(t)}\right] d t \sim \frac{-1}{\lambda} \int_{0}^{\infty} e^{-s t} \sum_{k=1}^{\infty} \frac{(-s \lambda l(t))^{k}}{k !} d t(45)
\end{aligned}
$$

which may be evaluated by further expanding $l(t), l^{2}(t), \ldots$ in powers of $t$ and using

$$
\int_{0}^{\infty} e^{-s t} t^{k} d t=\frac{\Gamma(k+1)}{s^{k+1}} .
$$

As an example, we present the motion starting with constant acceleration [25], for which $l(t)=\frac{1}{2} a t^{2}$ as $t \rightarrow 0$-where $a$ denotes the acceleration.

To the leading order (45) yields

$$
\begin{aligned}
\int_{0}^{\infty} e^{-s(\lambda \xi+\zeta(\xi))} d \xi & \sim s \int_{0}^{\infty} e^{-s t} l(t) d t=s \int_{0}^{\infty} e^{-s t} \frac{a t^{2}}{2} d t \\
& =\frac{a s}{2} s^{-3} \Gamma(3)=\frac{a}{s^{2}}
\end{aligned}
$$

so that for the first motion (i.e. $\lambda_{*}=\lambda_{0}$ ) we obtain

$$
\frac{\partial \hat{u}_{1}}{\partial x_{2}}\left(x_{1}, x_{2}, s\right) \sim \frac{a \cdot \Delta u E_{1}^{-}\left(\lambda_{0}\right) e^{s F\left(\lambda_{0}\right)}}{4 \sqrt{\pi} s^{3 / 2} F^{\prime \prime}\left(\lambda_{0}\right)^{1 / 2}} \quad \text { as } s \rightarrow \infty
$$

and

$$
\frac{\partial u_{1}}{\partial x_{2}}\left(x_{1}, x_{2}, t\right) \sim \frac{a \cdot \Delta u E^{-}\left(\lambda_{0}\right)}{2 \pi} \sqrt{t+F\left(\lambda_{0}\right)} H\left(t+F\left(\lambda_{0}\right)\right) \quad \text { as } t \rightarrow t_{0}^{+}=\left[-F\left(\lambda_{0}\right)\right]^{+} \text {. }
$$

The asymptotic behavior near the later wavefront can be discussed similarly with $\lambda_{*}=\lambda_{i}$, $i=1, \ldots, 5$. We have thus completed our study of the asymptotic wavefront behavior during the general nonuniform motion of an edge dislocation in a crystal when the dislocation axis is normal to a symmetry plane. The analysis presented here may also be useful for other moving singularities in anisotropic media.

\section{REFERENCES}

[1] A. W. Sáenz, J. Rat. Mech. Anal. 2 (1953), 83

[2] R. Bullough and B. A. Bilby, Proc. Phys. Soc. (London) 67B (1954), 615

[3] L. J. Teutonico, Phys. Rev. 124 (1961), 1039 
[4] J. D. Eshelby, Phys. Rev. 90 (1953), 248

[5] J. Weertman and J. R. Weertman, Dislocations in solids Vol. 3 (edited by F. R. N. Nabarro) North Holland, 1980

[6] X. Markenscoff and R. J. Clifton, J. Mech. Phys. Soc. 29 (1981), 253

[7] X. Markenscoff and R. J. Clifton, J. Appl. Mech. ASME 49 (1982), 792

[8] G. F. D. Duff, Phil. Trans. Roy. Soc. (London), 252A (1960), 249

[9] L. J. Teutonico, Mathematical theory of dislocations (edited by T. Mura), ASME, New York, 1969, 49

[10] J. D. Eshelby, Phil. Mag. 40 (1949), 903

[11] J. D. Eshelby, W. T. Read and W. Shockley, Acta Met 1 (1953), 251

[12] J. R. Willis, Phil. Mag., Sec. 8, 21 (1970), 931

[13] R. Burridge, Quart. J. Mech. Appl. Math. 23 (1970), 217

[14] A. T. de Hoop, Appl. Sci. Res., B8 (1960), 349

[15] R. Burridge, Quart. J. Mech. Appl. Math. 24 (1971), 81

[16] E. A. Kraut, Reviews of Geophysics 1 (1963), 401

[ 17] M. J. P. Musgrave, Crystal acoustics, Halden-Day Inc. 1970

[18] X. Markenscoff, J. Elast. 10 (1980), 193

[ 19] L. Knopoff and F. Gilbert, J. Acous. Soc. Amer. 31 (1959), 1161

[20] N. Bleinstein and R. A. Handelman, Asymptotic expansions of integrals, Holt, Reinhart \& Winston, New York, 1975

[21] W. L. Ferrar, Higher algebra, Oxford University Press, 1948

[22] L. B. Freund, Quart. Appl. Math. 30 (1972), 271

[23] D. V. Widder, The Laplace transform, Princeton University Press, 1946

[24] L. Sirovich, Techniques of asymptotic analysis, Springer-Verlag, New York, 1971

[25] X. Markenscoff, Int. J. Engng. Sci. 20 (1982), 289 